\title{
GPM Microwave Imager Design, Predicted Performance and Status
}

\section{David Newell, Ball Aerospace \& Technologies Corp. dnewellaball.com}

Sergey Krimchansky, NASA Goddard Space Flight Center

The Global Precipitation Measurement (GPM) Microwave Imager (GMI) Instrument is being developed by Ball Aerospace and Technology Corporation (BATC) for the GPM program at NASA Goddard.

The Global Precipitation Measurement (GPM) mission is an international effort managed by the National Aeronautics and Space Administration (NASA) to improve climate, weather, and hydro-meteorological predictions through more accurate and more frequent precipitation measurements. The GPM Microwave Imager (GMI) will be used to make calibrated, radiometric measurements from space at multiple microwave frequencies and polarizations. GMI will be placed on the GPM Core Spacecraft together with the Dualfrequency Precipitation Radar (DPR). The DPR is two-frequency precipitation measurement radar, which will operate in the $\mathrm{Ku}$-band and $\mathrm{Ka}$-band of the microwave spectrum. The Core Spacecraft will make radiometric and radar measurements of clouds and precipitation and will be the central element of GPM's space segment. The data products from GPM will provide information concerning global precipitation on a frequent, near-global basis to meteorologists and scientists making weather forecasts and performing research on the global energy and water cycle, precipitation, hydrology, and related disciplines. In addition, radiometric measurements from GMI and radar measurements from the DPR will be used together to develop a retrieval transfer standard for the purpose of calibrating precipitation retrieval algorithms. This calibration standard will establish a reference against which other retrieval algorithms using only microwave radiometers (and without the benefit of the DPR) on other satellites in the GPM constellation will be compared. 
The instrument has completed the Critical Design Review phase of the program. The design of the instrument is complete. We describe the instrument and predict the performance of the GMI instrument. During the CDR phase a few key requirements were matured. These are described and the instrument compliance to these updated requirements is shown. The center frequencies at $183 \mathrm{GHz}$ have been updated to match previous radiometers. In addition, the sine vibration environment has been updated based on information coming from the launch vehicle and spacecraft design.

The instrument interfaces have been finalized and the design completed. The final mechanical and electrical interfaces are described. The mechanical interface was specifically designed to provide isolation from the spacecraft and allow accommodation on the future low inclination spacecraft. An electrical interface was added coming from the spacecraft that allows the GMI integration to be blanked during Dual Precipitation Radar pulses. The implementation of this blanking is shown.

The instrument is currently in the flight production phase. Status and initial test results on the flight hardware is presented.

The GMI instrument is a conical-scanned passive microwave radiometer used to make calibrated, radiometric measurements from space at multiple microwave frequencies and polarizations from $10 \mathrm{GHz}$ to $190 \mathrm{GHz}$. The instrument has a 1.22 meter offset parabolic reflector for high spatial resolution and high beam efficiency. Receivers at each frequency receive, amplify and detect the microwave radiation with different detection approaches used at each frequency to maximize performance while minimizing cost and risk. Mechanical features include a deployment assembly for the main reflector and slip rings to transfer power and signals to the rotating portion of the instrument.

[1] Newell, D.A., D. Figgins, T. Ta, and B. Berdanier, "GPM microwave imager instrument design and predicted performance," IEEE Int. Geosci. Remote Sensing Symp. (IGARSS), pages 4426 - 4428, July 23-28, 2007. 
[2] Sechler, J.B., "GPM microwave imager selected calibration features and predicted performance," IEEE Int. Geosci. Remote Sensing Symp. (IGARSS), pages 5237 - 5239, July 23-28, 2007.

[3] S.W. Bidwell, G.M. Flaming, J.F. Durning, and E.A. Smith "The Global Precipitation Measurement (GPM) Microwave Imager (GMI) Instrument: Role, Performance, and Status" Int. Geosci. Remote Sensing Symp. (IGARSS) 2005

[4] Brown, S.T., S. Desai, Lu Wenwen, A.B. Tanner, "On the Long-Term Stability of Microwave Radiometers Using Noise Diodes for Calibration," IEEE Trans. Geosci.

Remote Sensing, vol. 45, no. 7, part 1, pages 1908-1920, May 2007. 